\title{
Outcomes reported in clinical studies of stable angina pectoris on traditional Chinese medicine: a systematic review towards a core outcome set
}

Mingyan Zhang ( $\sim$ zmy157@126.com)

Tianjin University of Traditional Chinese Medicine

Hui Zi Chua

Tianjin University of Traditional Chinese Medicine https://orcid.org/0000-0003-3927-9179

Bohan Niu

Tianjin University of Traditional Chinese Medicine

Wenke Zheng

Tianjin University of Traditional Chinese Medicine

\section{Fengwen Yang}

Tianjin University of Traditional Chinese Medicine

Xinyao Jin

Tianjin University of Traditional Chinese Medicine

Lishuang Zhang

Tianjin University of Traditional Chinese Medicine

Hongjie Zhao

Tianjin University of Traditional Chinese Medicine

\section{Rui Feng}

Tianjin University of Traditional Chinese Medicine

\section{Zhaochen Ji}

Tianjin University of Traditional Chinese Medicine

Haiyin Hu

Tianjin University of Traditional Chinese Medicine

\section{Kai Li}

Tianjin University of Traditional Chinese Medicine

Junhua Zhang ( $\nabla$ zjhtcm@foxmail.com )

Tianjin University of Traditional Chinese Medicine

\section{Boli Zhang}

Tianjin University of Traditional Chinese Medicine

\section{Research}

Keywords: Stable angina pectoris, Outcome measures, Core outcome set, clinical studies, Systematic review

Posted Date: February 23rd, 2021

DOI: https://doi.org/10.21203/rs.3.rs-219620/v1

License: (9) This work is licensed under a Creative Commons Attribution 4.0 International License. Read Full License 


\section{Abstract \\ Background}

Traditional Chinese medicine (TCM) plays an important role in contributing to the public health in China. However, nonstandardized outcomes in TCM related studies posed great difficulty in data synthesis for healthcare decisions. An agreed set of standardized outcomes, or termed as a core outcome set (COS), can solve this existing problem of outcome heterogeneity. This review aimed to analyze outcome heterogeneity, assess the quality of outcome reporting and providing a list of potential outcomes for COS development of SAP using TCM.

\section{Methods}

8 literature databases were searched to identify clinical studies about TCM for SAP (SAP-TCM) of yearly samples of 2005, 2010, 2015, 2016, 2018 and 2019. 2 clinical registry databases were searched from inception to October 2020. Outcomes definition, measurement methods and time-point were extracted and categorized into 7 themes which include clinical symptoms and signs, physical and chemical examination, TCM-related outcomes, adverse events, quality of life, long-term prognosis and economic evaluation. Timing and quality assessment of the outcomes reported were analyzed and evaluated.

\section{Results}

A total of 1166 studies were included from 12962 potential studies, involving 111426 patients. 231 outcomes were identified and a total of 89 outcomes reported at least twice in the studies. The most commonly reported theme was clinical symptoms and signs. The most frequently reported outcomes were total rate of ECG efficiency and angina attack in clinical studies of published literature and clinical registries databases respectively. Outcomes reported in a single study ranged from 1 to 15 , and 5 outcomes on average per study. $80 \%$ of the studies lack reporting of primary outcomes. A wide variation of measurement instruments or methods was used. A range of 1-17 measurement time-points were recorded. Quality assessment of outcome reporting was generally low, with $65.6 \%$ of the studies scoring 3 to 4 points.

\section{Conclusion}

Outcomes reported in studies on SAP-TCM are heterogeneous. A lack of standardization complicates comparisons of outcomes across studies. Developing a COS for SAP-TCM could contribute to the standardization of outcome reporting and offer a stronger evidence for health decision-making.

\section{Background}

Stable angina pectoris (SAP) is generally related to myocardial ischemia or hypoxia with the clinical manifestation of transient chest discomfort $^{1,2}$. In the majority of patients, the underlying mechanism is thought to be due to significant obstructive coronary artery disease ( $>70 \%$ diameter narrowing) of one or more vessels, which limits an increase in coronary blood flow during periods of increased myocardial oxygen demand during exertion ${ }^{1-6}$. The annual death rate of patients with SAP is 1$3 \%^{4-7}$. The usage of conventional antianginal drug therapy such as organic nitrates, $\beta$-blockers, calcium channel blockers, nicorandil, and ivabradine can effectively control angina and improve exercise tolerance ${ }^{8-10}$. However, there is limited evidence showing that the usage of conventional drug therapy can significantly improve adverse cardiac outcomes in patients with SAP clinically. Long term usage of these drugs contributing to side effects such as headaches and gastrointestinal ulcerations should not be neglected ${ }^{8}$.

Traditional Chinese medicine (TCM), being a part of complementary alternative medicine, plays an important role in contributing to the public health in China. The formation and development of TCM are mainly based on observations and 
experiences in long term clinical practices, whereby syndrome (pattern) differentiation acts as a basis in which TCM therapeutic principles are set upon, determined by using the four methods of diagnosis: tongue examination, history taking (inquiry), listening and smelling examination, palpation (pulse taking, abdominal examination, etc.). SAP is categorized by the TCM term"Xiongbr", comprising of 7 different TCM syndromes, namely blood stasis of the heart, Qi stagnation in the heart and chest, blockage of phlegm turbidity, cold coagulation in heart-vessel, heart and kidney yin deficiency, Qi and Yin deficiency, heart and kidney yang deficiency.

In recent years, there has been an increasing number of TCM related clinical trials in treating SAP to provide evidence for the efficacy and effectiveness of TCM ${ }^{11-15}$. Evidence-based approaches have significantly improved the quality of TCM clinical trials ${ }^{16}$. However, there are major challenges faced in the design of clinical trials, not only in TCM but also in modern medicine. One apparent drawback is the non-standardization of outcomes being chosen and assessed ${ }^{17-18}$. Heterogeneity of outcome reporting across studies posed as a challenge in completing meta-analyses and systematic reviews and caused studies to be excluded from the data syntheses. In addition, the definitions and measurement instruments of the same outcome are inconsistent across studies, which also poses difficulty in generating evidence to guide clinical practices and health care decisions. Hence, it is important to resolve the lack of methodological quality in clinical trials. The development of a core outcome set (COS) can help to address this problem ${ }^{19-21}$. A COS is an agreed standardized set of outcomes, which can be measured and reported, as a minimum, in all trials for the same healthcare area ${ }^{21}$. Developing a cos would minimize heterogeneity in outcome reporting, which will allow outcomes across studies to be combined and compared, enabling the results of clinical studies to address healthcare decisions and reduce research waste ${ }^{22}$. This COS study has been registered in Core Outcome Measures in Effectiveness Trials (COMET) website (available at http://www.comet-

initiative.org/studies/details/391).

The aim of this systematic review was to examine and determine the variations in outcome reporting, assessing the quality of outcome reporting as well as providing an overview of the types of outcomes reported in clinical studies regarding SAP using TCM. In addition, the review could provide a fundamental list of potentially relevant outcomes for COS development of SAP using TCM.

\section{Methods}

\section{Search Strategy}

We identified studies by performing a comprehensive electronic search strategy of 8 literature databases and 2 clinical registry databases. The databases are as followed:

1. 4 Chinese databases which include: China National Knowledge Infrastructure (CNKI), WanFang, VIP and SinoMed 2. 4 English databases which include: PubMed, Cochrane Library, Excepta Medica Database (Embase) and Web of Science 3. 2 clinical registry databases which include: Chinese Clinical Trial registry (ChiCTR)and ClinicalTrials.gov (NCT) ${ }^{23-24}$.

The initial search was conducted in October 2017, followed by an updated search in October 2020. Before the formal retrieval, we performed a pre-retrieval of the databases over a decade from 2005 to 2016 to get a rough idea of the workload in order to ensure the feasibility and scientificity of this study. However, it resulted in a large number of studies to be reviewed which appeared to be resource intensive, especially in literature databases. As we were interested in the changes and differences of outcomes over a period of time, we adjusted the retrieval time as follows:

1. For literature databases, we selected studies published in 2005, 2010, 2015 and 2016 as samples, and then added year 2018 and 2019 in the updated search. 
2. For the two clinical registry databases, we searched studies from inception to October 2017, and then updated from inception to October 2020.

The key terms used to guide the literature search such as 'stable angina pectoris', 'angina pectoris', 'chronic stable angina', 'chest pain', 'Xiongbi, 'clinical trial' and 'randomized controlled trial' combined as appropriate using the Boolean operands 'AND' and 'OR'. If Mesh terms or subject headings existed, they were included in the search. The TCM search term "Xiongbi" was inclusive of SAP and other cardiac diseases. Although the review focused on SAP, a broad search strategy was used to ensure that all potentially relevant articles were identified. Studies which include "Xiongbi" but not focused on SAP were subsequently excluded during eligibility assessment.

\section{Eligibility Criteria}

\section{(1) Inclusion criteria}

For inclusion in the review, a study had to meet the following criteria listed in Table 1.

Table 1

Inclusion criteria

\begin{tabular}{|ll|}
\hline Study type & Randomized controlled trials (RCTs), clinical controlled studies and case studies \\
\hline Population & People diagnosed with stable angina pectoris aged $\geq 18$ years \\
\hline Intervention & $\begin{array}{l}\text { 1) Any treatment of Chinese medicine such as decoction, patent medicine, extract and Chinese } \\
\text { medicine injection } \\
\text { 2) As adjuvant treatment, Chinese medicine with conventional western medicine }\end{array}$ \\
\hline $\begin{array}{l}\text { Control } \\
\text { intervention }\end{array}$ & No restriction \\
\hline Outcome & 1) Reporting at least one outcome in a study \\
\hline $\begin{array}{l}\text { Publication } \\
\text { status }\end{array}$ & 2) No restriction on the types of outcome. \\
\hline
\end{tabular}

\section{(2) Exclusion criteria}

The exclusion criteria for this study were as follows:

1. Studies which involved other complications.

2. Studies on acupuncture, Tuina and acupoint application as intervention measures or as combined interventions.

3. Studies not using human subject, review, dissertation, conference paper and duplicate studies.

4. Studies whereby full text could not be acquired.

Two reviewers (MZ and $\mathrm{HZC}$ ) independently screened the titles, abstracts, full-text papers and the registration information to identify studies. Any disagreement in the study selection was resolved by discussion with a third reviewer (JZ).

\section{Data extraction and analysis}


Two reviewers (MZ and HZC) independently screened all included studies and eligible studies were reviewed independently. Relevant data was extracted and cross-checked. Data was extracted using a pre-designed office access form. Any disagreement was resolved through discussion after a thorough reading of the paper or consulted with a third reviewer (JZ). Any missing information was supplemented by trying to contact the original author.

The following data were extracted from each included study:

1. Basic information: author(s); year of publication; organization; patient source

2. Target population baseline characteristics: number of recruited patients, patients' age, patients' sex, number of patients who withdrew from the study

3. Clinical characteristics: course of disease, severity of disease, TCM syndrome types

4. Intervention details: medication name, course of treatment, treatment frequency and dosage, length of follow-up

5. Outcome measure details: name of outcome, outcome measurement instruments or methods, time-point at which the outcome is being measured and outcome data classification.

Overlapping or duplicated outcomes were merged according to the definitions of outcomes and were categorized into 7 themes according to the developed taxanomy of outcome classification. Results were summarized using frequencies and percentages, and analyzed by descriptive analysis.

\section{Quality assessment of outcomes reported}

The quality of outcomes reported was being assessed after the selection of eligible studies. The quality assessment was conducted by scoring without weight division. Table 2 shows the criteria of the quality assessment, which was made according to other COS studies ${ }^{25-27}$.

Table 2

Seven items assessment of the reporting quality of outcome measures

\begin{tabular}{|c|c|c|c|}
\hline No. & Criterion & Yes & No \\
\hline 1 & Whether the outcome was clearly stated as primary or secondary outcome. & $\begin{array}{l}1 \\
\text { point }\end{array}$ & $\begin{array}{l}0 \\
\text { point }\end{array}$ \\
\hline 2 & $\begin{array}{l}\text { Whether the outcome was defined or not. Outcomes were considered defined if text of their } \\
\text { meaning or a citation was provided. }\end{array}$ & $\begin{array}{l}1 \\
\text { point }\end{array}$ & $\begin{array}{l}0 \\
\text { point }\end{array}$ \\
\hline 3 & $\begin{array}{l}\text { It was clearly described how the outcomes are measured or the outcome measurement (indicators } \\
\text { and/or tools used, if relevant). }\end{array}$ & $\begin{array}{l}1 \\
\text { point }\end{array}$ & $\begin{array}{l}0 \\
\text { point }\end{array}$ \\
\hline 4 & It was clearly described by whom the outcomes are measured. & $\begin{array}{l}1 \\
\text { point }\end{array}$ & $\begin{array}{l}0 \\
\text { point }\end{array}$ \\
\hline 5 & $\begin{array}{l}\text { It was clearly described the time points and time period at or during which outcome was } \\
\text { measured. }\end{array}$ & $\begin{array}{l}1 \\
\text { point }\end{array}$ & $\begin{array}{l}0 \\
\text { point }\end{array}$ \\
\hline 6 & $\begin{array}{l}\text { Are methods used to enhance the quality of outcome measurement (for example, repeated } \\
\text { measurement, training) if appropriate? }\end{array}$ & $\begin{array}{l}1 \\
\text { point }\end{array}$ & $\begin{array}{l}0 \\
\text { point }\end{array}$ \\
\hline 7 & $\begin{array}{l}\text { The reporting of outcomes was consistent throughout the article. There is no unambiguous } \\
\text { reporting that makes it confusing for the reader to assess what has been done. }\end{array}$ & $\begin{array}{l}1 \\
\text { point }\end{array}$ & $\begin{array}{l}0 \\
\text { point }\end{array}$ \\
\hline
\end{tabular}


A total of 12962 records were identified potentially eligible for inclusion from databases and clinical registries first searched in October 2017. The updated search in October 2020 retrieved another 8413 records, resulting in a total of 21375 records. After excluding duplicates, 16858 records were included in title and abstract screening. 7221 studies were preliminarily selected for full-text screening, with a total of 6055 studies excluded due to various factors such as review articles disease ambiguity, inclusion of unstable angina, non-drugs therapy, full text not being able to be obtained or of irrelevance. Finally, 1166 relevant studies were included for data extraction and analyses. Figure 1 shows the flow diagram of this systematic review.

\section{Characteristics of included studies}

Of the 1166 included studies, there was a majority of studies being RCTs (569/1166), with the other half of the studies being controlled studies and cross-sectional studies. All of the included studies were conducted in China. Apart from the 4 cohort studies in 211 cross-sectional studies, the rest were case reports. The sample size of the included studies varies from 18 to 3800 , with majority of the studies $(78.3 \%)$ having small sample sizes $(<100)$. Diagnosis of the severity of SAP using grade I-III according to the Canadian Cardiovascular Society (CCS) was preferred over New York Heart Association (NYHA) guideline and other guidelines set in China. There was only about $60 \%$ of the studies which reported on the duration of the disease.

A total of 805 studies showed reporting on TCM syndrome, which epidemiologically shows that blood stasis and phlegm are the two main TCM pathogenesis of SAP. The types of intervention used in the included studies were mainly used as an adjuvant therapy alongside conventional treatment or western medicine. TCM interventions included herbal decoction, Chinese patent medicine and intravenous TCM. A total of 1116 studies reported on the course of treatment, and the duration of the treatment varied from 7 days to 26 years. However, the course of treatment in majority of studies (93\%) was within three months, with a lack of long-term follow-up studies. Majority of the studies have reported obtaining either ethics approval or informed consent. The characteristics of all the included studies were shown in Table 3. 


\begin{tabular}{|c|c|c|c|}
\hline & $N(\%)$ & & $N(\%)$ \\
\hline Total included studies & $\begin{array}{l}1166 \\
(100)\end{array}$ & & \\
\hline Study type & & Types of intervention & \\
\hline Randomized controlled trials & $\begin{array}{l}569 \\
(48.8)\end{array}$ & $\mathrm{CM} / \mathrm{CM}+\mathrm{CT}$ & $\begin{array}{l}211 \\
(18.1)\end{array}$ \\
\hline Controlled trials & $\begin{array}{l}386 \\
(33.1)\end{array}$ & $\mathrm{CM}+\mathrm{CT}$ vs $\mathrm{CT} / \mathrm{CM}$ vs $\mathrm{CT}$ & $\begin{array}{l}571 \\
(49.0)\end{array}$ \\
\hline Cross-sectional studies & $\begin{array}{l}211 \\
(18.1)\end{array}$ & $\mathrm{CM}$ vs placebo/ CM + CT vs placebo $+\mathrm{CT}$ & $\begin{array}{l}64 \\
(5.5)\end{array}$ \\
\hline Study size & & $\begin{array}{l}\mathrm{CM} 1 \text { vs } \mathrm{CM} 2 / \mathrm{CM} 1+\mathrm{CT} \text { vs } \mathrm{CM} 2+\mathrm{CT} / \mathrm{CM} 1+\text { placebo } 2 \text { vs } \mathrm{CM} 2+ \\
\text { placebo1/ } \mathrm{CM} 1+\mathrm{CM} 2 \text { vs } \mathrm{CM} 2\end{array}$ & $\begin{array}{l}231 \\
(19.8)\end{array}$ \\
\hline $1-100$ & $\begin{array}{l}913 \\
(78.3)\end{array}$ & $\mathrm{CM}$ vs $\mathrm{PD} / \mathrm{CM}+\mathrm{CT}$ vs $\mathrm{PD}+\mathrm{CT} / \mathrm{CM}+\mathrm{PD}$ vs $\mathrm{PD}$ & $\begin{array}{l}89 \\
(7.6)\end{array}$ \\
\hline $100-200$ & $\begin{array}{l}229 \\
(19.7)\end{array}$ & Course of treatment & \\
\hline $200-300$ & $\begin{array}{l}11 \\
(0.9)\end{array}$ & $\leq 2 \mathrm{w}$ & $\begin{array}{l}256 \\
(22.0)\end{array}$ \\
\hline$\geq 300$ & $\begin{array}{l}13 \\
(1.1)\end{array}$ & $2-4 w$ & $\begin{array}{l}501 \\
(43.0)\end{array}$ \\
\hline Severity of disease & & $4-8 w$ & $\begin{array}{l}201 \\
(17.2)\end{array}$ \\
\hline Reported & $\begin{array}{l}559 \\
(47.9)\end{array}$ & $8-12 w$ & $\begin{array}{l}132 \\
(11.3)\end{array}$ \\
\hline $\operatorname{ccs}$ & $\begin{array}{l}336 \\
(28.8)\end{array}$ & $12 w$ & $\begin{array}{l}26 \\
(2.2)\end{array}$ \\
\hline NYHA & $\begin{array}{l}11 \\
(1.0)\end{array}$ & unclear & $\begin{array}{l}50 \\
(4.3)\end{array}$ \\
\hline $\begin{array}{l}\text { Guidelines for SAP treatment } \\
\text { developed in China }\end{array}$ & $\begin{array}{l}72 \\
(6.2)\end{array}$ & Ethics & \\
\hline Unclear & $\begin{array}{l}140 \\
(12.0)\end{array}$ & Reported & $\begin{array}{l}818 \\
(70.2)\end{array}$ \\
\hline Unreported & $\begin{array}{l}607 \\
(52.1)\end{array}$ & Informed consent & $\begin{array}{l}785 \\
(67.3)\end{array}$ \\
\hline Duration & & Ethical approval & $\begin{array}{l}160 \\
(13.7)\end{array}$ \\
\hline Reported & $\begin{array}{l}709 \\
(60.8)\end{array}$ & Unreported & $\begin{array}{l}348 \\
(29.8)\end{array}$ \\
\hline Unreported & $\begin{array}{l}457 \\
(39.2)\end{array}$ & Funding & \\
\hline TCM Syndrome & & Yes & $\begin{array}{l}212 \\
(18.2)\end{array}$ \\
\hline
\end{tabular}

Abbreviations: CCS, Canadian Cardiovascular Society; NYHA, New York Heart Association;CM, Chinese medicine, which mainly include herbal decoctions, Chinese patent medicine and intravenous TCM; CT, conventional therapy; PD, positive drug of western medicine; w, week. 


\begin{tabular}{|c|c|c|}
\hline & $N(\%)$ & $N(\%)$ \\
\hline Reported & $\begin{array}{l}805 \\
(69.0)\end{array}$ & $\begin{array}{l}954 \\
(81.8)\end{array}$ \\
\hline Blood stasis in the heart & $\begin{array}{l}343 \\
(29.4)\end{array}$ & \\
\hline $\begin{array}{l}\text { Blockage of phlegm turbidity in } \\
\text { chest and heart }\end{array}$ & $\begin{array}{l}257 \\
(22.0)\end{array}$ & \\
\hline Qi stagnation & $\begin{array}{l}102 \\
(8.7)\end{array}$ & \\
\hline deficiency in Qi and Yin & $\begin{array}{l}83 \\
(7.1)\end{array}$ & \\
\hline Cold coagulation in heart-vessel & $8(0.7)$ & \\
\hline Heart and kidney Yin deficiency & $8(0.7)$ & \\
\hline $\begin{array}{l}\text { Heart and kidney Yang } \\
\text { deficiency }\end{array}$ & $4(0.4)$ & \\
\hline Unreported & $\begin{array}{l}361 \\
(31.0)\end{array}$ & \\
\hline \multicolumn{3}{|c|}{$\begin{array}{l}\text { Abbreviations: CCS, Canadian Cardiovascular Society; NYHA, New York Heart Association;CM, Chinese medicine, which } \\
\text { mainly include herbal decoctions, Chinese patent medicine and intravenous TCM; CT, conventional therapy; PD, positive } \\
\text { drug of western medicine; w, week. }\end{array}$} \\
\hline
\end{tabular}

\section{List of outcomes assessed in included studies}

A total of 231 outcomes were reported in included studies of SAP using TCM treatment. 89 outcomes were reported at least twice in the studies and were further categorized into 7 themes, namely clinical symptoms and signs, physical and chemical examination, TCM-related outcomes, adverse events, long-term prognosis, quality of life and economic evaluation (Table 4). A variation of outcomes was reported across the 7 different themes. 49 outcomes $(55.1 \%, 49 / 89)$ reported were categorized in "physical and chemical examination" theme. The frequency of outcomes reported for the remaining themes include 12 outcomes $(13.5 \%, 12 / 89)$ for "long term prognosis", 9 outcomes $(10.1 \%, 9 / 89)$ for "clinical symptoms and signs", 9 outcomes for "adverse events", 6 outcomes $(6.7 \%, 6 / 89)$ for "TCM-related outcomes", 3 outcomes $(3.4 \%, 3 / 89)$ for "quality of life" and only 1 outcome $(1.1 \%, 1 / 89)$ for "economic evaluation".

\section{Measurement instruments/methods adopted to assess outcomes}

There was a lack of reporting for the outcome measurement instruments or its measurement methods in the included studies. Furthermore, there were different measurement methods for the same outcome and there was no consensus for the measurement standards.

A huge proportion of studies adopted self-made criteria, such as the pain degree measured during angina attack, the frequency and duration of angina attack. Seattle Angina Questionnaire (SAQ) was adopted as the main measurement method for quality of life (QoL) outcome in SAP patients. However, majority of the studies did not define the type of questionnaire that was used to assess QoL. Studies which merely reported using "a questionnaire" to assess QoL were listed as "undefined" in the measurement method.

\section{Timing of assessment for outcomes reported}


There was an average of 5 measurement time-points reported in studies, ranging from 1 to 17 time-points which the outcomes were being measured. The outcome 'adverse events or effects' has the highest frequency for measurement timepoint. 19 outcomes have 2 measurement timepoints.

A summary of the types of outcomes, the measurement instruments or methods used for individual outcomes as well as the number for different measurement timepoint for the included studies is shown in Table 4.

We summarized the timing of assessment for the 5 most frequently reported outcomes as shown in Table 5 . The most frequently reported outcomes in the included studies retrieved from published literature and clinical registry databases include total rate of ECG efficacy, total rate of angina efficacy, total rate of clinical efficacy, lipid profile test, effectiveness of TCM syndromes, effectiveness of TCM symptoms, angina attack, QoL-SAQ, exercise treadmill test (ETT) outcome and cardiovascular event. Timing of assessment for the outcomes were reported in the studies retrieved from publish literature and NCT. However, there was no reporting of outcome measurement timepoints in the studies retrieved from ChiCTR. Majority of the studies retrieved from publish literature assessed outcomes by efficacy rate, such as ECG efficacy rate, angina efficacy rate and clinical efficacy rate. 
Table 4

Outcomes reported in included studies of SAP on TCM

\begin{tabular}{|c|c|c|c|}
\hline Theme & Domain/outcomes & Measurement instruments/methods & $\begin{array}{l}\text { Measurement } \\
\text { time-point (n) }\end{array}$ \\
\hline CSS & Angina attack & & \\
\hline \multirow[t]{3}{*}{ CSS } & - Pain degree & 1 Score according to a certain or self-made criterion & 2 \\
\hline & & 2 Grading the severity(Normal, mild, moderate, severe) & \\
\hline & & 3 Visual analogue scale & \\
\hline \multirow[t]{4}{*}{ CSS } & - Frequency of angina attack & 1. Score according to a certain or self-made criterion & 7 \\
\hline & & 2. The number of attack per week & \\
\hline & & 3. Grading the severity(Normal, mild, moderate, severe) & \\
\hline & & 4. A Wearable Cardiac Monitor & \\
\hline \multirow[t]{3}{*}{ CSS } & - Duration of angina & 1. Score according to a certain or self-made criterion & 4 \\
\hline & & 2. Record time & \\
\hline & & 3. Grading the severity(Normal, mild, moderate, severe) & \\
\hline \multirow[t]{4}{*}{ cSS } & - Nitroglycerin administration & 1 Dosage and frequency of medication & 14 \\
\hline & & 2 Grading the severity(Normal, mild, moderate, severe) & \\
\hline & & $\begin{array}{l}3 \text { Withdrawal and decreasing rate of } \\
\text { nitroglycerin(withdrawal, reduction, unchanged) }\end{array}$ & \\
\hline & & 4 The amount of used short-acting nitroglycerin & \\
\hline \multirow[t]{2}{*}{ CSS } & Angina symptom score & 1 Undefined questionnaire & 11 \\
\hline & & 2 Score according to a certain or self-made criterion & \\
\hline \multirow[t]{5}{*}{ css } & Grading of changes in angina & $1 \mathrm{GCP}-\mathrm{TCM}$ & 4 \\
\hline & & 2 ECE* & \\
\hline & & $\begin{array}{l}3 \text { Guidelines of diagnostic and treatment on angina } \\
\text { pectoris }\end{array}$ & \\
\hline & & 4 Self-made criterion & \\
\hline & & $5 \mathrm{CCS}$ & \\
\hline CSS & Cardiac function grading & $\operatorname{ccs}$ & 2 \\
\hline
\end{tabular}

Abbreviations: CSS, clinical symptoms and signs; PCE, physical and chemical examination; TCMO, TCM-related outcomes; $A E$, adverse events; LTP,long-term prognosis; QoL, quality of life; $E E$, economic evaluation; $A P$, angina pectoris;ECG electrocardiography; $A E C G$, active electrocardiography; $S A Q$, seattle angina questionnaire; EQ-5D European five-dimensional health scale SF-36, 36-item short-form health survey; GQOL-74, generic quality of life inventory-74; SF-12, 12-item short-form health survey TED, total exercise duration; HRV, heart rate variability; 6MWT, six minute walking test; ETT, exercise treadmill test; METs, metabolic equivalents; TIB total ischemia burden; EF, ejection fraction; IMT intima-media thickness Vmax, maximum blood flow velocity; BAPWV, brachial-ankle pulse wave velocity; CTA, CT angiography; BNP, brain natriuretic peptide; VEGF, vascular endothelial growth factor; IL-1, interleukin-1; IL-6, interleukin-6; TNF-a, tumor necrosis factor a; CRP, c-reactive protein; hs-CRP, hypersensitive c-reactive protein; MCP-1, monocyte chemotactic protein 1; MMP-1, matrix metalloproteinase 1; sVCAM-1, soluble vascular cell adhesionmolecula-1; ET-1, endothelin-1; SOD superoxide dismutase; NO nitric oxide; NOS, nitric oxide synthase; vWF, von willebrand factor; Hcy, homocysteine; TXA2 thromboxane A2 TXB2, thromboxane B2; PT prothrombin time APTT activated partial thromboplastin time TT thrombin time FIB fibrinogen; GCP-TCM guiding principles of clinical research on new drugs of traditional Chinese medicine ${ }^{28}$ ECE$^{\star}$, the evaluation criteria of efficacy for angina pectoris of coronary heart disease and electrographic ${ }^{29}$; ELISA the enzyme-linked immunosorbent assay. 


\begin{tabular}{|c|c|c|c|}
\hline Theme & Domain/outcomes & Measurement instruments/methods & $\begin{array}{l}\text { Measurement } \\
\text { time-point (n) }\end{array}$ \\
\hline \multirow[t]{3}{*}{ CSS } & \multirow[t]{3}{*}{ Rate of clinical efficacy } & 1 Improvement of angina pectoris symptom & 11 \\
\hline & & 2 GCP-TCM & \\
\hline & & 3 Grading according to a certain or self-made criterion & \\
\hline \multirow[t]{2}{*}{ css } & \multirow{2}{*}{ Rate of angina efficacy } & 4 GCP-TCM & 9 \\
\hline & & 5 Grading according to a certain or self-made criterion & \\
\hline \multirow[t]{6}{*}{ TCMO } & \multicolumn{3}{|l|}{ TCM syndromes } \\
\hline & \multirow{2}{*}{$\begin{array}{l}\text { - Effectiveness of } \\
\text { TCM syndromes }\end{array}$} & 1 GCP-TCM & 14 \\
\hline & & 2 Grading according to a certain or self-made criterion & \\
\hline & \multirow[t]{3}{*}{ - TCM syndrome scoring } & 1 Undefined scale (s) & 15 \\
\hline & & 2 A self-made criterion & \\
\hline & & 3 The TCM syndrome scale of GCP-TCM & \\
\hline TCMO & TCM symptoms & & \\
\hline \multirow[t]{2}{*}{ TCMO } & \multirow{2}{*}{$\begin{array}{l}\text { - Effectiveness of TCM } \\
\text { symptoms }\end{array}$} & 1 GCP-TCM & 6 \\
\hline & & 2 Self-made criteria & \\
\hline
\end{tabular}

Abbreviations: CSS, clinical symptoms and signs; PCE, physical and chemical examination; TCMO, TCM-related outcomes;
AE, adverse events; LTP,long-term prognosis; QoL, quality of life; EE, economic evaluation; AP, angina
pectoris;ECG electrocardiography; AECG, active electrocardiography; SAQ, seattle angina questionnaire; EQ-5D European
five-dimensional health scale SF-36, 36-item short-form health survey; GQOL-74, generic quality of life inventory-74; SF-12,
12-item short-form health survey TED, total exercise duration; HRV, heart rate variability; 6MWT, six minute walking test; ETT,
exercise treadmill test; METs, metabolic equivalents; TIB total ischemia burden; EF, ejection fraction; IMT intima-media
thickness Vmax, maximum blood flow velocity; BAPWV, brachial-ankle pulse wave velocity; CTA, CT angiography; BNP,
brain natriuretic peptide; VEGF, vascular endothelial growth factor; IL-1, interleukin-1; IL-6, interleukin-6; TNF-a, tumor
necrosis factor a; CRP, C-reactive protein; hs-CRP, hypersensitive c-reactive protein; MCP-1, monocyte chemotactic protein 1;
MMP-1, matrix metalloproteinase 1; sVCAM-1, soluble vascular cell adhesionmolecula-1; ET-1, endothelin-1;
SOD superoxide dismutase; NO nitric oxide; NOS, nitric oxide synthase; vWF, von willebrand factor; Hcy, homocysteine;
TXA2 thromboxane A2 TXB2, thromboxane B2; PT prothrombin time APTT activated partial thromboplastin
time TT thrombin time FIB fibrinogen; GCP-TCM guiding principles of clinical research on new drugs of traditional
Chinese medicine ${ }^{28}$ ECE*, the evaluation criteria of efficacy for angina pectoris of coronary heart disease and
electrographic ${ }^{29}$; ELISA the enzyme-linked immunosorbent assay. 


\begin{tabular}{|c|c|c|c|}
\hline Theme & Domain/outcomes & Measurement instruments/methods & $\begin{array}{l}\text { Measurement } \\
\text { time-point (n) }\end{array}$ \\
\hline \multirow[t]{3}{*}{ TCMO } & \multirow[t]{3}{*}{ - TCM symptom scoring } & 1 GCP-TCM & \multirow[t]{3}{*}{9} \\
\hline & & 2 Undefined scale(s) & \\
\hline & & 3 A self-made criterion & \\
\hline \multirow[t]{4}{*}{ TCMO } & \multirow[t]{2}{*}{ Tongue } & 1 TCM inspection & \multirow[t]{2}{*}{1} \\
\hline & & 2 Grading by self-made criterion & \\
\hline & \multirow[t]{2}{*}{ Pulse } & 1 TCM palpation & \multirow[t]{2}{*}{1} \\
\hline & & 2 Grading by self-made criterion & \\
\hline \multirow[t]{2}{*}{ QoL } & \multirow[t]{2}{*}{ Quality of life scoring } & $1 \mathrm{EQ}-5 \mathrm{D}$ & \multirow[t]{2}{*}{12} \\
\hline & & $2 \mathrm{SAQ}$ & \\
\hline \multirow[t]{4}{*}{ QoL } & \multirow{4}{*}{$\begin{array}{l}\text { Improvement rate of life } \\
\text { quality }\end{array}$} & 3 SF-36 & \multirow[t]{4}{*}{11} \\
\hline & & 4 GQOL-74 & \\
\hline & & 5 SF-12 & \\
\hline & & 6 Undefined scale(s) & \\
\hline QoL & \multicolumn{2}{|l|}{ Sleep quality } & 1 \\
\hline LTP & \multicolumn{3}{|l|}{ Endpoint events } \\
\hline LTP & - All-cause mortality & Record the number of relevant events reported & 4 \\
\hline LTP & \multicolumn{2}{|l|}{ - Cardiogenic death } & 3 \\
\hline LTP & \multicolumn{2}{|l|}{ Cardiovascular event } & 3 \\
\hline LTP & \multicolumn{2}{|l|}{ - Myocardial infarction } & 2 \\
\hline LTP & \multicolumn{2}{|l|}{ - Stroke } & 1 \\
\hline LTP & \multicolumn{2}{|l|}{ - Unstable angina pectoris } & 1 \\
\hline LTP & \multicolumn{2}{|l|}{ - Coronary stent implantation } & 1 \\
\hline LTP & \multicolumn{2}{|l|}{ - Heart failure } & 2 \\
\hline LTP & \multicolumn{2}{|l|}{$\begin{array}{l}\text { - Incidence of severe } \\
\text { haemorrhages }\end{array}$} & 1 \\
\hline LTP & \multicolumn{2}{|l|}{$\begin{array}{l}\text { - Incidence of moderate } \\
\text { haemorrhages }\end{array}$} & 1 \\
\hline \multicolumn{4}{|c|}{$\begin{array}{l}\text { Abbreviations: CSS, clinical symptoms and signs; PCE, physical and chemical examination; TCMO, TCM-related outcomes; } \\
\text { AE, adverse events; LTP,long-term prognosis; QoL, quality of life; EE, economic evaluation; AP, angina } \\
\text { pectoris;ECG electrocardiography; AECG, active electrocardiography; SAQ, seattle angina questionnaire; EQ-5D European } \\
\text { five-dimensional health scale SF-36, 36-item short-form health survey; GQOL-74, generic quality of life inventory-74; SF-12, } \\
\text { 12-item short-form health survey TED, total exercise duration; HRV, heart rate variability; 6MWT, six minute walking test; ETT, } \\
\text { exercise treadmill test; METs, metabolic equivalents; TIB total ischemia burden; EF, ejection fraction; IMT intima-media } \\
\text { thickness Vmax, maximum blood flow velocity; BAPWV, brachial-ankle pulse wave velocity; CTA, CT angiography; BNP, } \\
\text { brain natriuretic peptide; VEGF, vascular endothelial growth factor; IL-1, interleukin-1; IL-6, interleukin-6; TNF-a, tumor } \\
\text { necrosis factor a; CRP, c-reactive protein; hs-CRP, hypersensitive c-reactive protein; MCP-1, monocyte chemotactic protein 1; } \\
\text { MMP-1, matrix metalloproteinase 1; sVCAM-1, soluble vascular cell adhesionmolecula-1;ET-1, endothelin-1; } \\
\text { SOD superoxide dismutase; NO nitric oxide; NOS, nitric oxide synthase; } \text { wWF, von willebrand factor; Hcy, homocysteine; } \\
\text { TXA2 thromboxane A2 TXB2, thromboxane B2; PT prothrombin time APTT activated partial thromboplastin } \\
\text { time TT thrombin time FIB fibrinogen; GCP-TCM guiding principles of clinical research on new drugs of traditional } \\
\text { Chinese medicine } 28 \text { ECE*, the evaluation criteria of efficacy for angina pectoris of coronary heart disease and } \\
\text { electrographic }{ }^{29} \text {; ELISA the enzyme-linked immunosorbent assay. }\end{array}$} \\
\hline
\end{tabular}




\begin{tabular}{|c|c|c|c|}
\hline Theme & Domain/outcomes & Measurement instruments/methods & $\begin{array}{l}\text { Measurement } \\
\text { time-point (n) }\end{array}$ \\
\hline LTP & Re-hospitalization & & 2 \\
\hline LTP & Clinic visits & & 1 \\
\hline \multirow[t]{2}{*}{ PCE } & ECG/AECG outcomes & 1 Holter system & \\
\hline & & 2 ECG & \\
\hline PCE & - ST segment depression & & 5 \\
\hline PCE & $\begin{array}{l}\text { - Flatness or inversion of } \mathrm{T} \\
\text { wave }\end{array}$ & & 4 \\
\hline PCE & $-\mathrm{TIB}$ & & 3 \\
\hline PCE & - HRV & & 2 \\
\hline \multirow[t]{2}{*}{ PCE } & - Total rate of ECG efficiency & 1 GCP-TCM & 11 \\
\hline & & 2 Self-made criteria & \\
\hline PCE & 6MWT outcomes & & \\
\hline PCE & - Walking distance & 6MWT & 3 \\
\hline PCE & $\begin{array}{l}\text { - Myocardial oxygen } \\
\text { consumption }\end{array}$ & & 2 \\
\hline PCE & ETT outcomes & & \\
\hline PCE & - Time to onset of chest pain & 1. Bruce protocol of ETT & 4 \\
\hline PCE & - Time to $1 \mathrm{~mm}$ ST-depression & 2. Modified Bruce protocol of ETT & 4 \\
\hline PCE & - Total exercise duration (TED) & & 10 \\
\hline PCE & - The Duke treadmill score & & 2 \\
\hline PCE & - Maximal heart rate & & 1 \\
\hline PCE & - METs & & 4 \\
\hline PCE & - Total of ST-depression & & 1 \\
\hline PCE & $\begin{array}{l}\text { - Number of leads with ST- } \\
\text { depression } \geqq 1 \mathrm{~mm}\end{array}$ & & 2 \\
\hline PCE & $\mathrm{EF}$ & Echocardiography & 2 \\
\hline PCE & Carotid ultrasound outcome & & \\
\hline
\end{tabular}

Abbreviations: CSS, clinical symptoms and signs; PCE, physical and chemical examination; TCMO, TCM-related outcomes; $A E$, adverse events; LTP,long-term prognosis; QoL, quality of life; $E E$, economic evaluation; AP, angina pectoris;ECG electrocardiography; $A E C G$, active electrocardiography; $S A Q$, seattle angina questionnaire; EQ-5D European five-dimensional health scale SF-36, 36-item short-form health survey; GQOL-74, generic quality of life inventory-74; SF-12, 12-item short-form health survey TED, total exercise duration; HRV, heart rate variability; 6MWT, six minute walking test; ETT, exercise treadmill test; METs, metabolic equivalents; TIB total ischemia burden; EF, ejection fraction; IMT intima-media thickness Vmax, maximum blood flow velocity; BAPWV, brachial-ankle pulse wave velocity; CTA, CT angiography; BNP, brain natriuretic peptide; VEGF, vascular endothelial growth factor; IL-1, interleukin-1; IL-6, interleukin-6; TNF-a, tumor necrosis factor a; CRP, c-reactive protein; hs-CRP, hypersensitive c-reactive protein; MCP-1, monocyte chemotactic protein 1; MMP-1, matrix metalloproteinase 1; sVCAM-1, soluble vascular cell adhesionmolecula-1; ET-1, endothelin-1; SOD superoxide dismutase; NO nitric oxide; NOS, nitric oxide synthase; vWF, von willebrand factor; Hcy, homocysteine; TXA2 thromboxane A2 TXB2, thromboxane B2; PT prothrombin time APTT activated partial thromboplastin time TT thrombin time FIB fibrinogen; GCP-TCM guiding principles of clinical research on new drugs of traditional Chinese medicine ${ }^{28}$ ECE$^{\star}$, the evaluation criteria of efficacy for angina pectoris of coronary heart disease and electrographic ${ }^{29}$; ELISA the enzyme-linked immunosorbent assay. 


\begin{tabular}{|c|c|c|c|}
\hline Theme & Domain/outcomes & Measurement instruments/methods & $\begin{array}{l}\text { Measurement } \\
\text { time-point (n) }\end{array}$ \\
\hline PCE & - IMT & \multirow[t]{2}{*}{ Carotid ultrasound } & 2 \\
\hline PCE & - Vmax & & 2 \\
\hline PCE & BAPWV & Ultrasound & 3 \\
\hline PCE & CTA coronary stenosis & CTA & 2 \\
\hline PCE & Lipid profile test & \multirow[t]{4}{*}{ General laboratory tests } & 14 \\
\hline PCE & Hemorheology indexes & & 8 \\
\hline PCE & BNP & & 1 \\
\hline PCE & VEGF & & 2 \\
\hline PCE & Blood glucose & & 3 \\
\hline PCE & Myocardial enzymes & & 2 \\
\hline PCE & Troponin & & 2 \\
\hline PCE & Inflammatory factors & & \\
\hline PCE & $-\mathrm{IL}-1$ & \multirow[t]{8}{*}{ ELISA } & 1 \\
\hline PCE & $-\mathrm{IL}-6$ & & 4 \\
\hline PCE & - TNF-a & & 5 \\
\hline PCE & - CRP & & 5 \\
\hline \multirow[t]{4}{*}{ PCE } & - hs-CRP & & 7 \\
\hline & - MCP-1 & & 2 \\
\hline & - MMP-1 & & / \\
\hline & - sVCAM-1 & & 2 \\
\hline PCE & - Cystatin c & General laboratory tests & / \\
\hline PCE & $-S O D$ & & 1 \\
\hline \multirow[t]{2}{*}{ PCE } & - Visfatin & & 1 \\
\hline & Endothelial injury outcome & & / \\
\hline PCE & $-\mathrm{ET}-1$ & & 3 \\
\hline
\end{tabular}

Abbreviations: CSS, clinical symptoms and signs; PCE, physical and chemical examination; TCMO, TCM-related outcomes; $A E$, adverse events; LTP,long-term prognosis; QoL, quality of life; $E E$, economic evaluation; AP, angina pectoris;ECG electrocardiography; $A E C G$, active electrocardiography; $S A Q$, seattle angina questionnaire; EQ-5D European five-dimensional health scale SF-36, 36-item short-form health survey; GQOL-74, generic quality of life inventory-74; SF-12, 12-item short-form health survey TED, total exercise duration; HRV, heart rate variability; 6MWT, six minute walking test; ETT, exercise treadmill test; METs, metabolic equivalents; TIB total ischemia burden; EF, ejection fraction; IMT intima-media thickness Vmax, maximum blood flow velocity; BAPWV, brachial-ankle pulse wave velocity; CTA, CT angiography; BNP, brain natriuretic peptide; VEGF, vascular endothelial growth factor; IL-1, interleukin-1; IL-6, interleukin-6; TNF-a, tumor necrosis factor a; CRP, c-reactive protein; hs-CRP, hypersensitive c-reactive protein; MCP-1, monocyte chemotactic protein 1; MMP-1, matrix metalloproteinase 1; sVCAM-1, soluble vascular cell adhesionmolecula-1; ET-1, endothelin-1; SOD superoxide dismutase; NO nitric oxide; NOS, nitric oxide synthase; vWF, von willebrand factor; Hcy, homocysteine; TXA2 thromboxane A2 TXB2, thromboxane B2; PT prothrombin time APTT activated partial thromboplastin time TT thrombin time FIB fibrinogen; GCP-TCM guiding principles of clinical research on new drugs of traditional Chinese medicine ${ }^{28}$ ECE$^{\star}$, the evaluation criteria of efficacy for angina pectoris of coronary heart disease and electrographic ${ }^{29}$; ELISA the enzyme-linked immunosorbent assay. 


\begin{tabular}{|c|c|c|c|}
\hline Theme & Domain/outcomes & Measurement instruments/methods & $\begin{array}{l}\text { Measurement } \\
\text { time-point (n) }\end{array}$ \\
\hline PCE & $-\mathrm{NO}$ & & 2 \\
\hline \multirow[t]{2}{*}{ PCE } & - NOS & & \\
\hline & $-v W F$ & & 1 \\
\hline PCE & - Hcy & & 9 \\
\hline PCE & Coagulation outcomes & & \\
\hline PCE & - TXA2 & & 0,4 \\
\hline PCE & $-\mathrm{TXB2}$ & & \\
\hline PCE & - Platelet aggregation & & 6 \\
\hline PCE & $\begin{array}{l}\text { - Four items of coagulation } \\
\text { test (PT,APTT,TT,FIB) }\end{array}$ & & 5 \\
\hline PCE & Neurotransmitter & & 3 \\
\hline EE & Health economic evaluation & \multirow[t]{2}{*}{ Unreported } & \\
\hline EE & - Medical cost & & / \\
\hline $\mathrm{AE}$ & $\begin{array}{l}\text { General safety outcomes } \\
\text { monitoring }\end{array}$ & \multirow{8}{*}{$\begin{array}{l}1 \text { Grading the safety degree according to a certain } \\
\text { or self-made criterion } \\
2 \text { General laboratory tests }\end{array}$} & \\
\hline $\mathrm{AE}$ & - Vital signs & & 6 \\
\hline $\mathrm{AE}$ & - Urine routine & & 12 \\
\hline$A E$ & - Blood routine & & 13 \\
\hline $\mathrm{AE}$ & - Stool routine & & 8 \\
\hline$A E$ & - Liver function & & 14 \\
\hline$A E$ & - Renal function & & 14 \\
\hline$A E$ & - Electrolyte & & 4 \\
\hline$A E$ & Adverse events/effects & Record related events & 17 \\
\hline$A E$ & QT interval of ECG & ECG & 5 \\
\hline \multicolumn{4}{|c|}{$\begin{array}{l}\text { Abbreviations: CSS, clinical symptoms and signs; PCE, physical and chemical examination; TCMO, TCM-related outcomes; } \\
\text { AE, adverse events; LTP,long-term prognosis; QoL, quality of life; EE, economic evaluation; AP, angina } \\
\text { pectoris;ECG electrocardiography; AECG, active electrocardiography; SAQ, seattle angina questionnaire; EQ-5D European } \\
\text { five-dimensional health scale SF-36, 36-item short-form health survey; GQOL-74, generic quality of life inventory-74; SF-12, } \\
\text { 12-item short-form health survey TED, total exercise duration; HRV, heart rate variability; 6MWT, six minute walking test; ETT, } \\
\text { exercise treadmill test; METs, metabolic equivalents; TIB total ischemia burden; EF, ejection fraction; IMT intima-media } \\
\text { thickness Vmax, maximum blood flow velocity; BAPWV, brachial-ankle pulse wave velocity; CTA, CT angiography; BNP, } \\
\text { brain natriuretic peptide; VEGF, vascular endothelial growth factor; IL-1, interleukin-1; IL-6, interleukin-6; TNF-a, tumor } \\
\text { necrosis factor a; CRP, c-reactive protein; hs-CRP, hypersensitive c-reactive protein; MCP-1, monocyte chemotactic protein 1; } \\
\text { MMP-1, matrix metalloproteinase } 1 ; \text { sVCAM-1, soluble vascular cell adhesionmolecula-1; ET-1, endothelin-1; } \\
\text { SOD superoxide dismutase; NO nitric oxide; NOS, nitric oxide synthase; vWF, von willebrand factor; Hcy, homocysteine; } \\
\text { TXA2 thromboxane A2 TXB2, thromboxane B2; PT prothrombin time APTT activated partial thromboplastin } \\
\text { time TT thrombin time FIB fibrinogen; GCP-TCM guiding principles of clinical research on new drugs of traditional } \\
\text { Chinese medicine } 28 \text { ECE*, the evaluation criteria of efficacy for angina pectoris of coronary heart disease and } \\
\text { electrographic }{ }^{29} \text {; ELISA the enzyme-linked immunosorbent assay. }\end{array}$} \\
\hline
\end{tabular}


Table 5

Measurement time-point of the 5 most frequently reported outcomes

\begin{tabular}{|c|c|c|c|c|c|c|}
\hline \multirow[t]{2}{*}{ Rank } & \multicolumn{2}{|c|}{ Published studies } & \multicolumn{2}{|c|}{ Protocols in ChiCTR (n) } & \multicolumn{2}{|c|}{ Protocols in NCT (n) } \\
\hline & Outcome & Time-points & Outcome & $\begin{array}{l}\text { Time- } \\
\text { points }\end{array}$ & Outcome & Time-points \\
\hline \multirow[t]{12}{*}{1} & \multirow{12}{*}{$\begin{array}{l}\text { ECG efficacy } \\
\text { rate }\end{array}$} & $10 \mathrm{~d}$ & \multirow{12}{*}{$\begin{array}{l}\text { Angina } \\
\text { attack }\end{array}$} & \multirow[t]{12}{*}{ Unreported } & \multirow[t]{12}{*}{ Angina attack } & $0,2 w ;$ \\
\hline & & $0,2 w$ & & & & $0,4 w$ \\
\hline & & $0,3 w$ & & & & $0,6 w$ \\
\hline & & $0,4 w$ & & & & $0,8 w$ \\
\hline & & $0,6 w$ & & & & $0,12 w ;$ \\
\hline & & $0,8 w$ & & & & $0,6 \mathrm{~m}$ \\
\hline & & $0,12 w$ & & & & $0,1 w, 2 w, 4 w, 8 w, 12 w$ \\
\hline & & $0,4 w, 8 w$ & & & & $0,2 w, 4 w, 8 w ;$ \\
\hline & & $0,1 w, 2 w, 3 w, 4 w$ & & & & $-2 w, 0 w, 6 w, 12 w$ \\
\hline & & \multirow[t]{3}{*}{$0,2 w, 4 w, 6 w, 8 w, 10 w, 12 w$} & & & & $-2 w, 0 w, 4 w, 8 w, 12 w ;$ \\
\hline & & & & & & $-2 w, 0 w, 12 w ;$ \\
\hline & & & & & & $0 \sim 14 \mathrm{~d}, 43$ 57d; \\
\hline \multirow[t]{7}{*}{2} & \multirow{7}{*}{$\begin{array}{l}\text { Angina } \\
\text { efficacy rate }\end{array}$} & $0,2 w$ & \multirow{7}{*}{$\begin{array}{l}\text { Quality of } \\
\text { life-SAQ }\end{array}$} & \multirow[t]{7}{*}{ Unreported } & \multirow[t]{7}{*}{ ETT outcomes } & $0,4 \mathrm{w}$ \\
\hline & & $0,3 w$ & & & & $0,57 \mathrm{~d}$ \\
\hline & & $0,4 w$ & & & & $0,4 w, 8 w$ \\
\hline & & $0,6 w$ & & & & $0,2 w, 4 w, 6 w ;$ \\
\hline & & $0,8 w$ & & & & $-2 w, 0,6 w ;$ \\
\hline & & $0,12 w ;$ & & & & $-2 w, 0,12 w$ \\
\hline & & $0,2 w, 4 w, 8 w$ & & & & \\
\hline \multirow[t]{6}{*}{3} & \multirow{6}{*}{$\begin{array}{l}\text { Clinical } \\
\text { efficacy rate }\end{array}$} & $0,2 w$ & \multirow{6}{*}{$\begin{array}{l}\text { ECG efficacy } \\
\text { rate }\end{array}$} & \multirow[t]{6}{*}{ Unreported } & \multirow{6}{*}{$\begin{array}{l}\text { ECG efficacy } \\
\text { rate }\end{array}$} & $0,4 \mathrm{w}$ \\
\hline & & $0,4 w$ & & & & $0,8 w$ \\
\hline & & $0,3 w$ & & & & $1 \mathrm{w}, 8 \mathrm{w}$ \\
\hline & & $0,12 w$ & & & & $0,4 w, 8 w$ \\
\hline & & \multirow[t]{2}{*}{$0,6 \mathrm{~m}$} & & & & $0,1 w, 2 w, 4 w, 0,8 w, 12 w ;$ \\
\hline & & & & & & $-2 w, 0,12 w$ \\
\hline
\end{tabular}

Abbreviations: ECG, electrocardiography; ETT, exercise treadmill test

* "0" refers to baseline. 


\begin{tabular}{|c|c|c|c|c|c|c|}
\hline \multirow{3}{*}{$\begin{array}{l}\text { Rank } \\
4\end{array}$} & \multicolumn{2}{|c|}{ Published studies } & \multicolumn{2}{|c|}{ Protocols in ChiCTR (n) } & \multicolumn{2}{|c|}{ Protocols in NCT (n) } \\
\hline & \multirow{11}{*}{$\begin{array}{l}\text { Lipid profile } \\
\text { test }\end{array}$} & 10d; & \multirow{11}{*}{$\begin{array}{l}\text { Effectiveness } \\
\text { of TCM } \\
\text { symptoms }\end{array}$} & \multirow[t]{11}{*}{ Unreported } & \multirow{11}{*}{$\begin{array}{l}\text { Quality of life- } \\
\text { SAQ }\end{array}$} & $0,4 w$ \\
\hline & & $0,2 w ;$ & & & & $0,12 \mathrm{w}$ \\
\hline & & $0,3 w$ & & & & $0,4,8 w$ \\
\hline & & $0,4 w$ & & & & $0,6 \mathrm{~m}$ \\
\hline & & $0,6 w$ & & & & $0,1 w, 2 w, 4 w, 8 w, 12 w ;$ \\
\hline & & $0,8 w$ & & & & $0,2 w, 4 w, 8 w, 12 w ;$ \\
\hline & & $0,12 w ;$ & & & & $-2 \mathrm{w}, 0 \mathrm{w}, 4 \mathrm{w}, 8 \mathrm{w}, 12 \mathrm{w}$ \\
\hline & & $0,6 \mathrm{~m}$ & & & & \\
\hline & & $0,9 \mathrm{~m}$ & & & & \\
\hline & & $0,4 w, 12 w$ & & & & \\
\hline & & $0,4 w, 8 w, 12 w ;$ & & & & \\
\hline \multirow[t]{8}{*}{5} & \multirow{8}{*}{$\begin{array}{l}\text { Effectiveness } \\
\text { of TCM } \\
\text { syndromes }\end{array}$} & $0,2 w$ & \multirow{8}{*}{$\begin{array}{l}\text { Lipid profile } \\
\text { test }\end{array}$} & \multirow[t]{8}{*}{ Unreported } & \multirow{8}{*}{$\begin{array}{l}\text { Cardiovascular } \\
\text { event }\end{array}$} & $0,57 \mathrm{~d}$ \\
\hline & & $0,3 w$ & & & & $2 \mathrm{w}, 4 \mathrm{w}$ \\
\hline & & $0,4 w$ & & & & $0,4 w, 8 w, 12 w$ \\
\hline & & $0,6 w$ & & & & $90 d$ \\
\hline & & $0,8 w$ & & & & \\
\hline & & $0,12 w$ & & & & \\
\hline & & $0,2 w, 4 w, 8 w$ & & & & \\
\hline & & $0,4 w, 12 w$ & & & & \\
\hline \multicolumn{7}{|c|}{ Abbreviations: ECG, electrocardiography; ETT, exercise treadmill test } \\
\hline \multicolumn{7}{|c|}{ * "0" refers to baseline. } \\
\hline
\end{tabular}

\section{Variations of outcomes reported among studies}

There was a wide variation in the number of outcomes reported across studies(Fig. 2). For studies published in literature databases, the number of outcomes reported in a single study ranged from 1 to 15, with an average of 5 outcomes per study (Fig. 2). 61.6\% (693/1124) of the studies reported 5 or lesser outcomes. For studies retrieved from clinical registry databases, the number of outcomes reported in a single study ranged from 1 to 35. Half of the studies (21/42) reported 6 to 10 outcomes and there were 2 studies with none reported. It is noteworthy that the outcomes were defined as primary or secondary in all of the studies retrieved from clinical registry databases. However, there is no definition of primary and secondary outcomes in $80 \%$ of the studies retrieved from published literature databases.

The proportion of outcomes reported in the studies retrieved from published literature databases and from clinical registry databases were almost identical across the 7 themes. Clinical signs and symptoms were the most frequently reported outcomes (33.3\%, 2167/6510, Fig. 3A; 44.8\%, 161/359, Fig. 3B) and the least reported outcomes belonged to economic evaluation ( $0 \%$, Fig. 3A; $1.4 \%, 5 / 359$, Fig. 3B). The most to least reported outcomes categorized into the different themes are as follow: clinical signs and symptoms, physical and chemical examination, TCM related outcomes, adverse events, quality of life, long term prognosis and lastly economic evaluation (Fig. 3). 


\section{Quality assessment of outcomes reported}

The quality assessment of outcomes reported in more than half of the included studies $(65.6 \%, 765 / 1166)$ scored from 3-4, which showed that the reporting quality of outcomes were generally low. More than half of the studies had a scoring of 3 or less $(63.1 \%, 763 / 1166)$, with the exception of a small percentage of studies with a score of $5(5.7 \%, 66 / 1166)$ for the quality assessment of outcomes reported (Fig. 4)

More than $80 \%$ of the studies retrieved from published literature databases did not clearly state or define primary or secondary outcomes. Although there was reporting on the measurement time-points for outcome assessment in the included studies, there was incomplete reporting in "how to measure" and "who to measure". Nearly all the studies retrieved from the clinical registry databases clearly stated primary or secondary outcomes. However, prior to the reporting on outcome measurements such as measurement methods and measurement time-points, studies retrieved from NCT were clearly described and more concise than those retrieved from ChiCTR.

\section{Discussion}

This systematic review is the first to demonstrate the heterogeneity in outcome reporting, as well as the inconsistency in measurement instruments and time-points used in outcome assessment for clinical trials of SAP using TCM. This review also categorizes the different types of outcomes and measurement instruments used to assess the efficacy and effectiveness of TCM treatments for SAP and evaluated the quality assessment of outcomes reporting. It is evident that the complexity of outcome assessment and the heterogeneity in the outcomes reported existed in clinical trials of SAP, be it in the field of TCM or conventional medicine. The inability for heterogenous outcomes to be compared and combined for meta-analyses deters results in studies to be utilized for clinical guidance and healthcare decision making, leading to a large amount of research waste $^{30-31}$. Standardized outcomes that matter most to different stakeholders is necessary ${ }^{32-33}$.

We did a comprehensive search of electronic databases which include four English and four Chinese published literature databases, as well as one English and one Chinese clinical registry databases. We included both Chinese and English language publications, hence there is no issue for selection bias. As the included clinical studies only involve adults, the results are not applicable to children and adolescents which is rarely seen in them. Also, the intervention was restricted to TCM (mainly oral Chinese medicine and intravenous TCM), all included studies were carried out by researchers from China.

This review included clinical trials as well as clinical controlled studies and case reports, which contribute to the greater range of outcomes collected. However, outcomes reported in these studies reflected a lack of consistency and standardization. There was much more emphasis on outcomes in relevance to clinical signs and symptoms $(33.3 \%, 2167 / 6510$, Fig. 3A) as well as physical and chemical examinations $(27.9 \%, 1816 / 6510$, Fig. $3 A)$, but limited reporting of long-term outcomes $(0.6 \%, 36 / 6510$, long term prognosis, Fig. $3 \mathrm{~A})$ and patient-oriented outcomes $(5.5 \%, 359 / 6510$, quality of life, Fig. $3 \mathrm{~A})$. It is necessary that outcomes should highly represent the perspective and need of relevant stakeholders, such as quality of life, which is an assessment for patient-reported outcomes (PROs) ${ }^{34-35}$. However, as evidenced in this review, PROs have received limited attention with only 35 of the 1124 included studies retrieved from published literature reported on QoL measure. On an encouraging note, the proportion was higher for QoL reporting in studies retrieved from clinical registry databases (33.3\%, 14/42), which reflected studies from clinical registries placed PROs on a higher emphasis. The duration for follow up for outcome assessment in NCT registered studies was also significantly longer than other studies and an increased reporting in outcomes of cardiovascular events (closely related to prognosis of SAP). This reflected a higher clinical relevance and importance of the outcomes reported in the studies retrieved from clinical registry databases.

Outcomes deemed to be important and practical will only exist when these outcomes truly reflect the need and perspectives of relevant stakeholders ${ }^{35}$. Apart from the perspectives of patients, clinicians or healthcare professionals is also one of the stakeholders necessary for COS development ${ }^{31,35-37}$. Our study revealed that though the treadmill exercise test and 6-minute walk test are outcomes which matter the most to clinicians, there were only $28.5 \%(332 / 1166)$ and $23.1 \%(269 / 1166)$ included 
studies which reported the outcomes respectively. Of which, the most commonly reported outcome in the registered studies retrieved from NCT was the treadmill exercise test, which was less frequently reported in registered studies retrieved from ChiCTR. This could be due to the lack of methodological quality and quality control of registered studies in ChiCTR, resulting in incomplete or unclear definition of outcome reporting as well as outcomes which do not fully reflect the perspective of the relevant stakeholders.

TCM related outcomes were reported more often in clinical studies retrieved from publish literature $(21.3 \%, 1384 / 6510$, Fig. 3A) and significantly lesser in studies retrieved from clinical registry protocols (8.4\%, 30/259, Fig. 3B). However, it is evident that studies using TCM interventions had limited reporting on TCM related outcomes, and even not reported in some included studies. This will result in few TCM related outcomes being collected and cannot fully represent the characteristics and advantages of TCM treatment.

Similarly, inconsistency in outcome reporting was seen across 1166 included studies, with inadequate reporting or absence in defining primary or secondary outcomes. About $80 \%$ of the published literature studies $(n=1124)$ did not define primary or secondary outcomes. Though all of the studies of clinical trial registries $(n=42)$ define primary and secondary outcomes, there was inadequate reporting about "when" and "how" the outcomes should be measured. This review highlighted an important lack in clarity in the reporting of outcome measurement methods, with inaccurate representation and definition of outcomes being assessed. Some studies reported translating continuous to categorical variables when assessing the outcomes reported. For instance, outcomes on angina efficacy, ECG efficacy, clinical efficacy and TCM syndrome efficacy were translated to hierarchical data such as highly effective, significantly effective and not effective. This translation from continuous to categorical variables used in assessment of outcome reporting dilutes the original data collected and severely affect the value and utilization of research results.

Timing of assessment for outcomes reported was relatively short, majority of the studies reported assessing outcomes in 2 to 4 weeks. The short timeframe in the assessment of outcomes could be contributed by the consideration of challenges in long term research or funding issues. Short term outcomes can be much easily assessed and measured. However, the limited reporting of long-term outcomes such as long-term prognosis and cardiovascular death or adverse events will not reflect the true need for the clinicians, patients or policy makers, resulting in the lack of practicality or utilization in future clinical studies.

A wide variation in the measurement instruments were used for the same outcome and certain studies did not mention any measurement instruments or methods used in outcome assessment. For instance, in studies measuring PROs, many questionnaires used for assessment were poorly defined or explained. As evidently shown in this review, the majority of included studies used scoring scales as an assessment instrument or method for TCM related outcomes and clinical symptoms. These scoring scales were mainly subjective and not standardized, or even self-made without any reference backing. This limited the interpretability of the assessed outcomes as it is unknown whether validated measurement instruments were used. Furthermore, it will hinder the quality of generating a preliminary list of outcomes for cos development which could result in no TCM related outcomes to be included in the final COS. Standardizing of "what to measure" is also required to assess certain outcomes, especially in cases when similar outcomes were being assessed, which will improve cross-studies comparisons and data analyses.

Our study has some limitations that need to be addressed. To limit the workload and the practicality for this review due to the high volume of literature needed to be reviewed, we included only studies with Chinese herbs or medicine related interventions and exclude studies with acupuncture or Tuina interventions, as well as confining to certain years in databases search. This might result in certain types of potential outcomes being neglected. Moreover, the scope of this review used the nomenclature of western medicine, neglecting the nomenclature of TCM syndromes. Characteristics and representativeness of TCM related outcomes were also limited as this review analysed on the outcomes reported in clinical studies of SAP on TCM rather than clinical studies of Xiongbi on TCM. However, we need to consider that despite SAP belongs to the TCM term Xiongbi, Xiongbi encompasses other diseases such as myocardial infarction and atherosclerosis which makes it difficult for the outcomes reported in the studies of different diseases to be clearly defined. Additionally, TCM syndrome is complex and hard to define, 
especially when different TCM syndromes will arise due to the different body constitutions of the patients in various countries or states.

By seeking to develop a COS, problems arising from heterogeneity of outcome reporting and the standardisation or clarity of outcome measurement methods or instruments will be addressed. The development of COS in SAP studies for TCM will reduce reporting bias in outcomes and provide guidance towards standardisation of outcome reporting in future SAP-TCM clinical studies. The use of a COS will also serve to support future data synthesis and meta-analysis across studies, providing guidance to relevant stakeholders on outcomes and measurement instruments or methods which matter most to them. This is especially vital for the development of evidence-based TCM which COS development serve as a reference for standardization of outcomes in TCM clinical studies. Furthermore, COS serves to improve the quality in TCM related clinical studies by implementing a robust and sound methodology in order to achieve practical results for the effectiveness or efficacy of TCM to be recognized.

\section{Conclusion}

This review highlights the non-standardization of outcome reporting in clinical studies of SAP-TCM and the lack of clarity in the definitions or descriptions of the outcomes reported, including their measurement methods and instruments used in assessment. The importance of developing a COS for clinical studies in SAP-TCM will help in reducing the heterogeneity reporting of outcomes and improving the quality assessment of outcomes to support data synthesis of clinical studies for SAP-TCM.

\section{List Of Abbreviations}

SAP: Stable angina pectoris; TCM: Traditional Chinese medicine; SAP-TCM: Stable angina pectoris on Traditional Chinese Medicine; COS: Core outcome set; COMET: Core Outcome Measures in Effectiveness Trials; CNKI: China National Knowledge Infrastructure; ChiCTR: Chinese Clinical Trial registry; NCT: ClinicalTrials.gov; CCS: Canadian Cardiovascular Society; NYHA: New York Heart Association; SAQ: Seattle Angina Questionnaire; QoL: Quality of life; ETT: Exercise treadmill test; PROs: Patientreported outcomes.

\section{Declarations}

\section{Ethics approval and consent to participate}

Not applicable

\section{Consent for publication}

Not applicable.

\section{Availability of data and materials}

Not applicable

\section{Competing interests}

All authors declare that they have no competing interests.

\section{Funding}

This work was supported by the National Natural Science Foundation of China (No.81473544, No.81904056).

\section{Authors' contributions}


$\mathrm{MZ}$ and $\mathrm{JZ}$ designed the study. $\mathrm{MZ}$ and $\mathrm{HZC}$ wrote the paper and conceived the study. $\mathrm{HZC}$ contributed the English translation of this protocol. MZ, HZC, BN and WZ carried out the literature search. XJ, LZ, HZ, RF, ZJ and HH contributed in screening and data extraction for literature search. MZ, HZC, BN and KL contributed to the quality assessment of outcome reporting and analysed the data extracted. JZ and BZ contributed to the overall supervision of the study. All authors read and approved the final manuscript.

\section{Acknowledgments}

Not applicable.

\section{References}

1. Montalescot G, Sechtem U, Achenbach S, et al. 2013 ESC guidelines on the management of stable coronary artery disease: the Task Force on the management of stable cor- onary artery disease of the European Society of Cardiology. Eur Heart J. 2013;34:2949-3003.

2. Opie LH. Angina pectoris: the evolution of concepts. J Cardiovasc Pharmacol Ther. 2004;9(Suppl 1):3-9.

3. Fraker TD, Finn ST, et al. 2007 chronic angina focused update of the ACC/AHA 2002 guidelines for the management of patients with chronic stable angina. Circulation. 2007;116:2762-72.

4. Fihn SD, Garlin JM, Abrams J, et al. ACCF/AHA/ACP/AATS/ PCNA/SCAI/STS guidelines for the diagnosis and management of patients with stable ischemic heart disease. Circulation. 2012;126:e354-471.

5. Fihn SD, Blankenship JC, Alexander KP, et al. 2014 ACCF/AHA/ ACP/AATS/PCNA/SCAI/STS focused update of the guidelines for the diagnosis and management of patients with stable ischemic heart disease. Circulation. 2014;130:174967.

6. Mancini GBJ, Gosselin G, Chow B, et al. Canadian cardiovascular society guidelines for the diagnosis and management of stable is- chemic heart disease. Can J Cardiol. 2014;30:837-49.

7. Thadani U. The pursuit of optimum outcomes in stable angina. Am J Cardiovasc Drugs 2003; (Suppl 1); pp 11-20.

8. Thadani U. Management of patients with chronic stable angina at low risk for serious cardiac events. Am J Cardiol. 1997;80:421-5.

9. Thadani U. Management of Stable Angina - Current Guidelines: A Critical Appraisal. Cardiovasc Drugs Ther. 2016;30(4):419-26.

10. Ambrosio G, Mugelli A, Lopez-Sendón J, Tamargo J, Camm J. Management of stable angina: A commentary on the European Society of Cardiology guidelines [published correction appears in Eur J Prev Cardiol. 2017 May;24(7):NP4]. Eur J Prev Cardiol. 2016;23(13):1401-1412.

11. Sun MY, Miao Y, Jin M, et al. Effect and Safety of Guanxinning Tablet for Stable Angina Pectoris Patients with Xin (Heart)Blood Stagnation Syndrome: A Randomized, Multicenter, Placebo-Controlled Trial. Chin J Integr Med. 2019;25(9):684-90.

12. Bi YF, Mao JY, Wang XL, et al. Study on syndrome differentiation and treatment in the management of chronic stable coronary artery disease to improve quality of life [published correction appears in Medicine (Baltimore). 2019 Apr;98(16):e15401]. Medicine (Baltimore). 2018;97(36):e12097.

13. Zhang ZF, Xu FQ, Liu HX, et al. A multicenter, randomized, double-blind clinical study on Wufuxinnaoqing Soft Capsule in treatment of chronic stable angina patients with blood stasis syndrome. Chin J Integr Med. 2015;21(8):571-8.

14. Wang Y, Zhang Y, Du Y, et al. A multicenter, randomized, double-blind, placebo-controlled trial evaluating the efficacy and safety of Taoren Honghua Jian granule in patients with stable coronary artery disease. Med (Baltim). 2019;98(44):e17753.

15. Yi G, et al., The usefulness of xuefu zhuyu tang for patients with angina pectoris: a meta-analysis and systematic review. Evidence-based complementary and alternative medicine: eCAM, 2014. 2014: p. 521602-521602.

16. Zhang MY, Yang FW,Zhang JH. Quality evaluation of randomized controlled clinical trials in Chinese Journal of Integrated Traditional and Western Medicine[J]. Chinese Journal of Evidence-Based Medicine,2017,17(03):357-363. 
17. Wu J, et al. Sini decoction as an adjuvant therapy for angina pectoris: a systematic review of randomized controlled trials. J Tradit Chin Med. 2017;37(1):12-22.

18. Zhang Z, Zhang. Outcome Measures of Clinical Trials of Stable Angina Pectoris Treated by Traditional Chinese Medicine in 2005: Literature Research[J]. Chinese Journal of Integrated Traditional and Western Medicin,2018,38(02):191-197.

19. Zhang L, et al., Clinical research of traditional chinese medicine needs to develop its own system of core outcome sets. Evidence-based complementary and alternative medicine: eCAM, 2013. 2013: p. 202703-202703.

20. Williamson P, Althma D, Blazeby J, Clarke M, Gargon E. Driving up the quality and relevance of research through the use of agreed core outcomes. J Health Serv Res Policy. 2012;17:1-2.

21. Williamson PR, et al. Developing core outcome sets for clinical trials: issues to consider. Trials. 2012;13:132.

22. Chan AW, et al. Increasing value and reducing waste: addressing inaccessible research. Lancet. 2014;383(9913):257-66.

23. Chinese Clinical Trials Registry. http://www.chictr.org.cn.

24. ClinicalTrials.gov https://clinicaltrials.gov.

25. Harman NL, Bruce IA, Callery P, et al. MOMENT-Management of Otitis Media with Effusion in Cleft Palate: protocol for a systematic review of the literature and identification of a core outcome set using a Delphi survey. Trials. 2013;14:70.

26. Alkhaffaf B, Blazeby JM, Williamson PR, et al. Reporting of outcomes in gastric cancer surgery trials: a systematic review. BMJ Open. 2018;8:e21796.

27. Heemskerk SCM, Rotteveel AH, Melenhorst J, Breukink SO, Kimman ML, Dirksen CD. Heterogeneous outcome reporting in adult slow-transit constipation studies: Systematic review towards a core outcome set. J Gastroenterol Hepatol. 2020;35(2):192-203.

28. Zheng XY. Guiding principles of clinical research on new drugs of traditional Chinese medicine[M]. Beijing: China Medical Science Press; 2002. pp. 85-6.

29. The evaluation criteria of efficacy for. angina pectoris of coronary heart disease and electrographic((Symposium on the Treatment of Coronary Heart Disease, Angina Pectoris and Arrhythmia with Integrated Traditional Chinese and Western Medicine, 1979, Shanghai))[J]. Chinese Pharmaceutical Affairs,1987,(02):17-20.

30. Gargon E, Gorst SL, Williamson PR. Choosing important health outcomes for comparative effectiveness research: 5th annual update to a systematic review of core outcome sets for research. PLoS One. 2019 Dec 12;14(12):e0225980.

31. Williamson PR, Altman DG, Bagley H, Barnes KL, Blazeby JM, Brookes ST, et al. The COMET Handbook: version 1.0. Trials. 2017;18(Suppl 3):280. Epub 2017/07/07.

32. Zhang MY, Zhang JH, Zhang BL.Outcome measures of clinical trials of stable angina pectoris treated by Chinese medicine in 2005: literature research[J].China Medical Abstracts(Internal Medicine),201835(02):73.

33. Gargon E, Williamson PR, Blazeby JM, Kirkham JJ. Improvement was needed in the standards of development for cancer core outcome sets. J Clin Epidemiol. 2019. Epub 2019/04/23.

34. Rivera SC, Kyte DG, Aiyegbusi OL, Slade AL, McMullan C, Calvert MJ. The impact of patient-reported outcome (PRO) data from clinical trials: a systematic review and critical analysis. Health Qual Life Outcomes. 2019 Oct;16(1):156. 17 (.

35. Kirkham JJ, Davis K, Altman DG, Blazeby JM, Clarke M, Tunis S, et al. Core Outcome Set-STAndards for Development: The COS-STAD recommendations. PLoS Med. 2017;14(11):e1002447. Epub 2017/ 11/18.

36. Kirkham JJ, Gorst S, Altman DG, Blazeby JM, Clarke M, Devane D, et al. Core Outcome Set-STAndards for Reporting: The COS-STAR Statement. PLoS Med. 2016;13(10):e1002148. Epub 2016/10/19.

37. Kirkham JJ, Gorst S, Altman DG, Blazeby JM, Clarke M, Tunis S, et al. Core Outcome Set-STAndardised Protocol Items: the COS-STAP Statement. Trials. 2019;20(1):116.

\section{Figures}




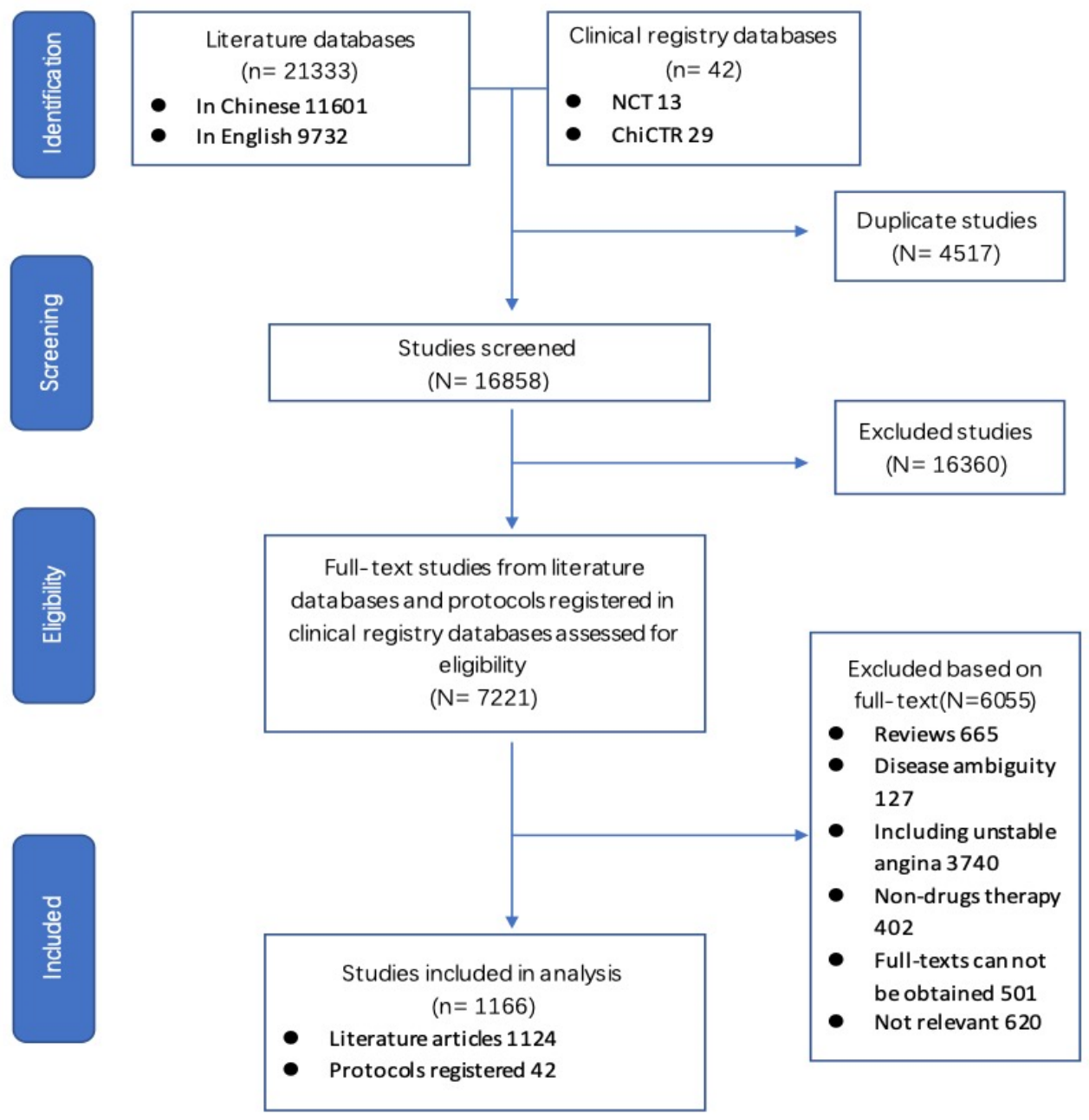

\section{Figure 1}

Flow diagram of study selection 


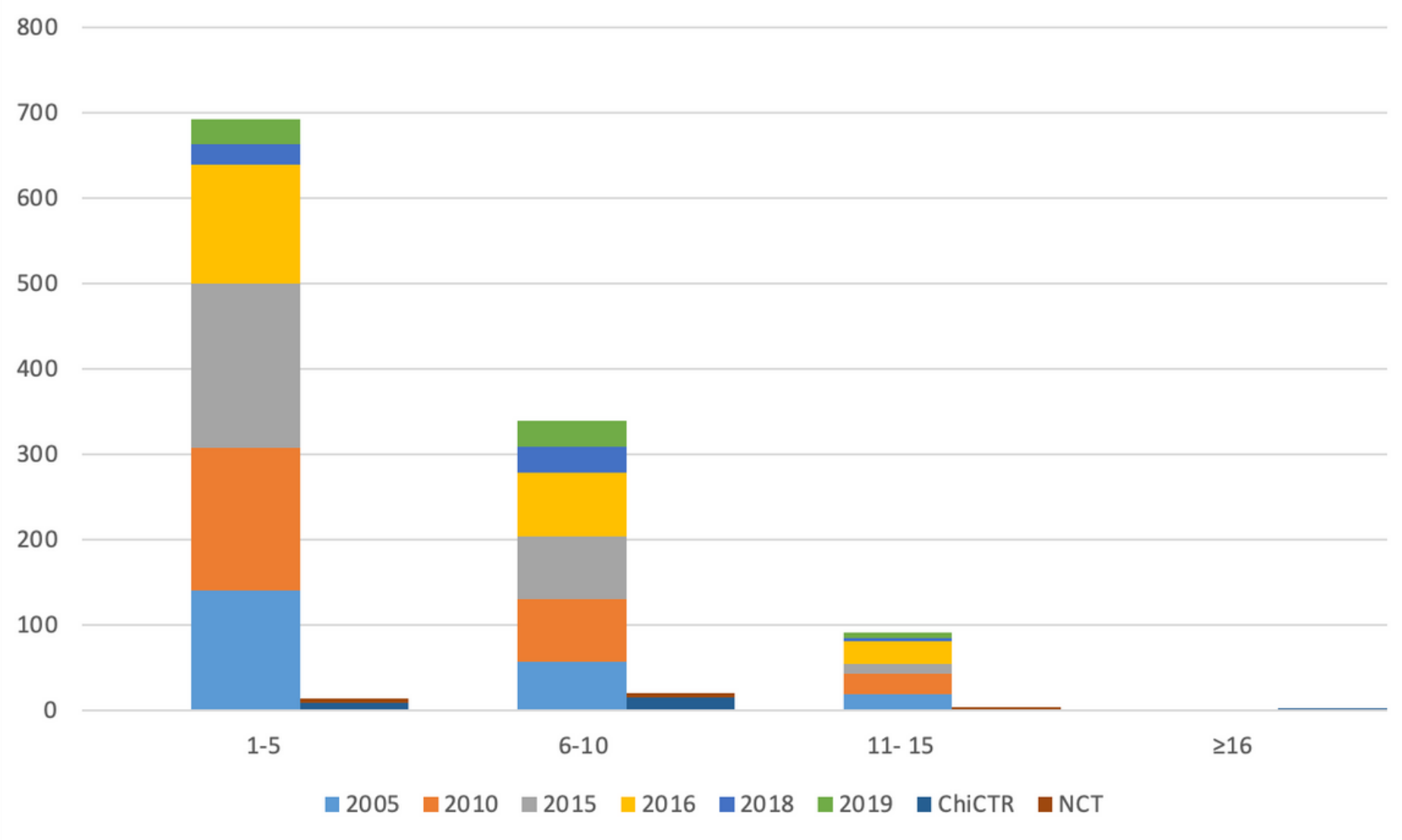

Figure 2

Quantity of outcomes reported in the included studies 

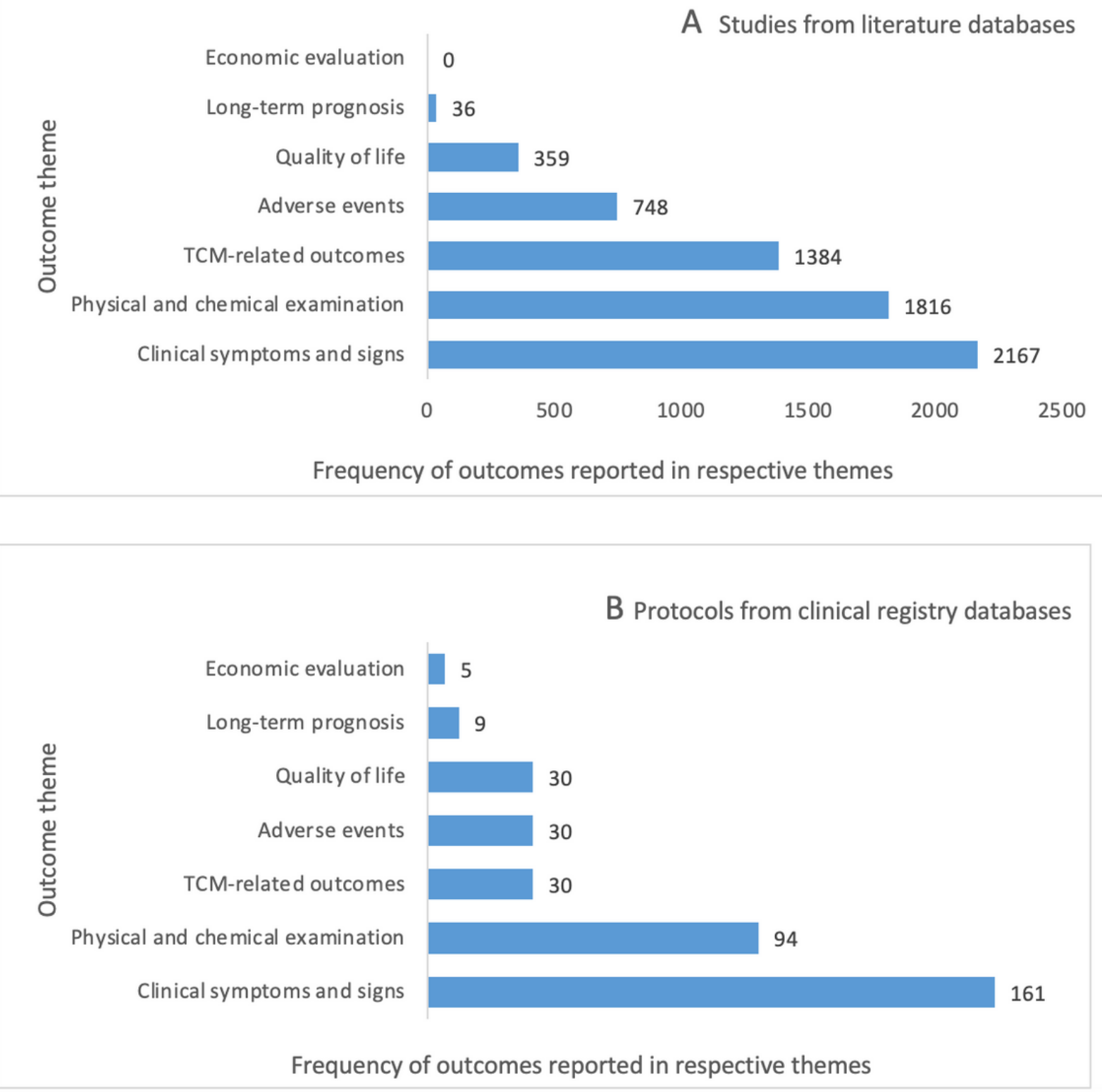

\section{Figure 3}

Frequency of outcomes reported in respective domains in SAP studies on TCM from (A) published literature databases and (B) clinical registry databases 


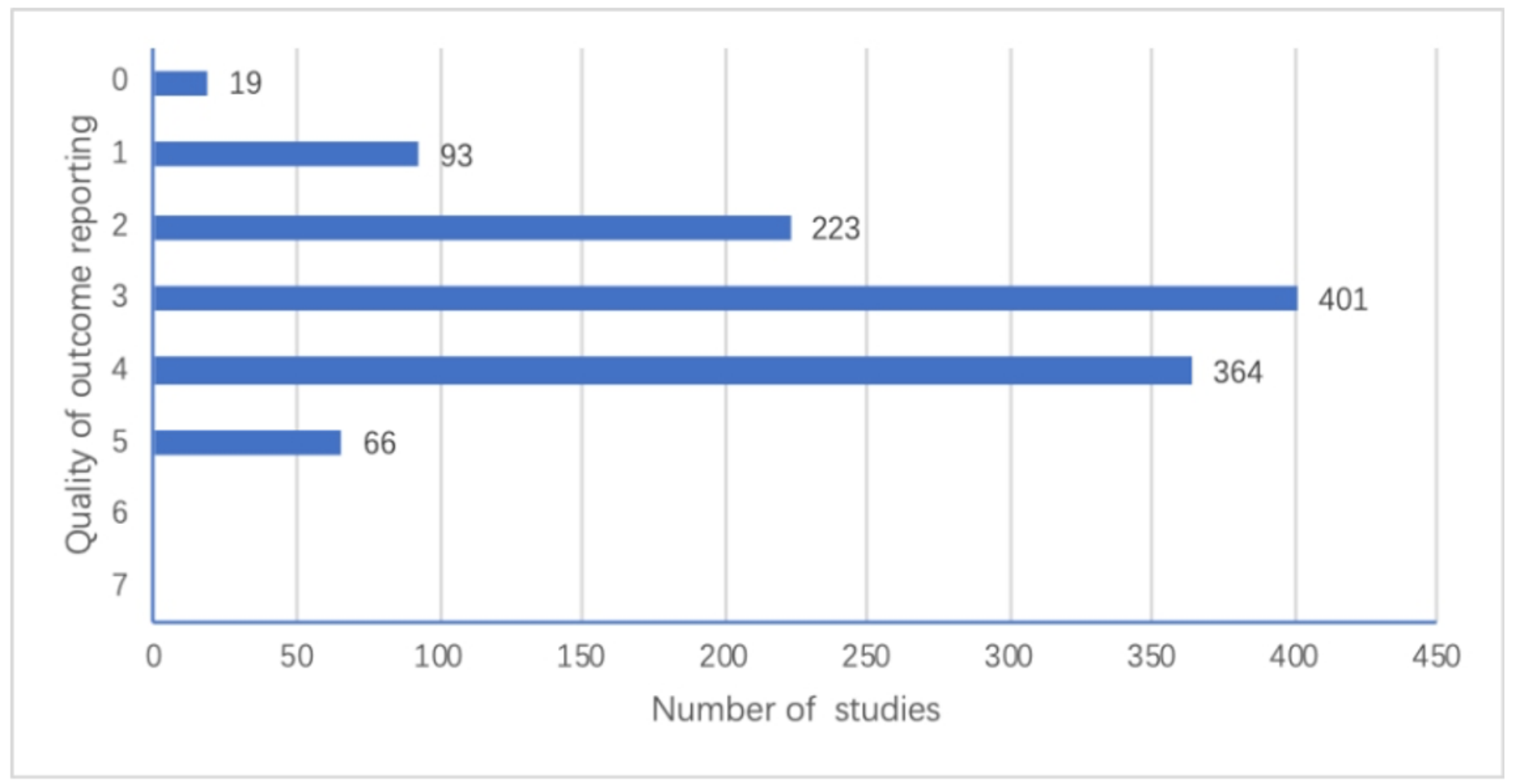

Figure 4

Quality assessment of outcomes reported in included studies 